\title{
Qualification and Integration Aspects of the DSSC Mega-Pixel X-Ray Imager
}

\author{
K. Hansen ${ }^{\circledR}$, Senior Member, IEEE, H. Klär, P. Kalavakuru ${ }^{\circledR}$, C. Reckleben, A. Venzmer, E. Wüstenhagen, \\ R. Schappeit, O.-C. Zeides, F. Okrent, C. Wunderer, M. Lemke, H. Graafsma, I. Schlosser, \\ M. Manghisoni ${ }^{\circledR}$, Senior Member, IEEE, E. Riceputi, S. Aschauer, L. Strüder, J. Soldat, \\ M. Tangl, F. Erdinger, A. Kugel ${ }^{\circledR}$, Member, IEEE, P. Fischer, L. Andricek, J. Ninković, \\ M. Turcato, and M. Porro, Member, IEEE
}

\begin{abstract}
The focal-plane module is the key component of the DEPFET sensor with signal compression (DSSC) mega-pixel $\mathrm{X}$-ray imager and handles the data of $128 \times 512$ pixels. We report on assembly-related aspects, discuss the experimental investigation of bonding behavior of different adhesives, and present the metrology and electrical test results of the production. The module consists of two silicon ( $\mathrm{Si}$ ) sensors with flip-chip connected CMOS integrated circuits, a Si-heat spreader, a low-temperature co-fired ceramics circuit board, and a molybdenum frame. A low-modulus urethane-film adhesive fills the gaps between onboard components and frame. It is also used between board and heat spreader, reduces the misfit strain, and minimizes the module warpage very efficiently. The heat spreader reduces the on-board temperature gradient by about one order of magnitude. The placement precision of the bare modules to each other and the frame is characterized by a standard deviation below 10 and $65 \mu \mathrm{m}$, respectively. The displacement due to the in-plane rotation and vertical tilting errors remains below 80 and $50 \mu \mathrm{m}$, respectively. The deflection of the sensor plane shows a mean value below $30 \mu \mathrm{m}$ with a standard deviation below $15 \mu \mathrm{m}$. Less than $4 \%$ of the application-specified integrated circuits (ASICs) exhibit a malfunction. More than two-thirds of the sensors have a maximum leakage current below $1 \mu \mathrm{A}$.
\end{abstract}

Index Terms-Adhesive strength, assembly, circuit testing, hybrid integrated circuits, image sensor, metrology, packaging technology, X-ray detector.

Manuscript received June 21, 2019; accepted July 5, 2019. Date of publication July 8, 2019; date of current version August 15, 2019. This work was supported by the European XFEL GmbH in the framework of the DSSC Project.

K. Hansen, H. Klär, P. Kalavakuru, C. Reckleben, A. Venzmer, E. Wüstenhagen, R. Schappeit, O.-C. Zeides, F. Okrent, C. Wunderer, M. Lemke, and H. Graafsma are with Deutsches Elektronen-Synchrotron DESY, 22607 Hamburg, Germany (e-mail: karsten.hansen@ desy.de).

I. Schlosser is with AEMtec GmbH, 12489 Berlin, Germany.

M. Manghisoni and E. Riceputi are with the Dipartimento di Ingegneria e Scienze Applicate, Università degli studi di Bergamo, 24129 Bergamo, Italy.

S. Aschauer and L. Strüder are with PNSensor GmbH, 81739 Munich, Germany.

J. Soldat, M. Tangl, F. Erdinger, A. Kugel, and P. Fischer are with the Institute of Computer Engineering, Heidelberg University, 69117 Heidelberg, Germany.

L. Andricek and J. Ninković are with the Semiconductor Laboratory of the Max-Planck Society, 81739 Munich, Germany.

M. Turcato and M. Porro are with European XFEL, 22869 Schenefeld, Germany.

Color versions of one or more of the figures in this paper are available online at http://ieeexplore.ieee.org.

Digital Object Identifier 10.1109/TNS.2019.2927421

\section{INTRODUCTION}

$\mathbf{T}$ HE DEPFET sensor with signal compression (DSSC) $\mathrm{X}$-ray imager for experiments at the European $\mathrm{X}$-ray free-electron laser (XFEL) Facility is being developed [1]. The DEPFET sensor is a symbiosis of DEPFET and silicon (Si)-drift detector (SDD) type drift rings [2]. For fast signalcharge collection, the pixel has a hexagonal shape with two drift rings and a side length of $136 \mu \mathrm{m}$. The sensor has a thickness of $450 \mu \mathrm{m}$ and is fully depleted for the detection of single photons in the soft X-ray regime from 0.5 to about $6 \mathrm{keV}$ at frame rates of up to $4.5 \mathrm{MHz}$. A unique feature of the mega-pixel camera is the pixel-level 8-bit digitization, the digital storage capability of up to 800 frames and the powerpulsing capability [3]. The current camera version is based on miniaturized (mini-SDD) cells with the first amplifying transistor in the readout application-specified integrated circuit (ASIC) and provides a linear transfer characteristic. For the single-photon resolution, a slope of at least one photon per analog-to-digital converter (ADC) bin is needed. In order to minimize the pixel-error rate, the ADC permits a precise trimming of the gain and offset [4]. The influence of gain and offset errors, as well as noise and differential nonlinearity on the experiment-relevant image fidelity, is treated in [4] and [5]. The conceptual design of the camera-head electronics was already presented in [6]. The focal-plane module (FPM) represents the key component of the imager. This paper focuses on the FPM design, describes the technology-related assembly aspects, and summarizes the results of the production of the first mini-SDD based camera.

In Section II, the construction of the camera head with its FPMs and interconnection to the peripheral electronics is presented. Section III summarizes the results of preliminary studies on materials and processes which are needed to set up the FPMs. In Section IV, the applicability of the finally chosen processes is evaluated. Section V presents the results of the series production of the FPMs.

\section{CAMERA HEAD}

\section{A. Quadrant}

Fig. 1 shows a photograph of a single quadrant of the mini-SDD camera. The quadrant-camera head comprises four 

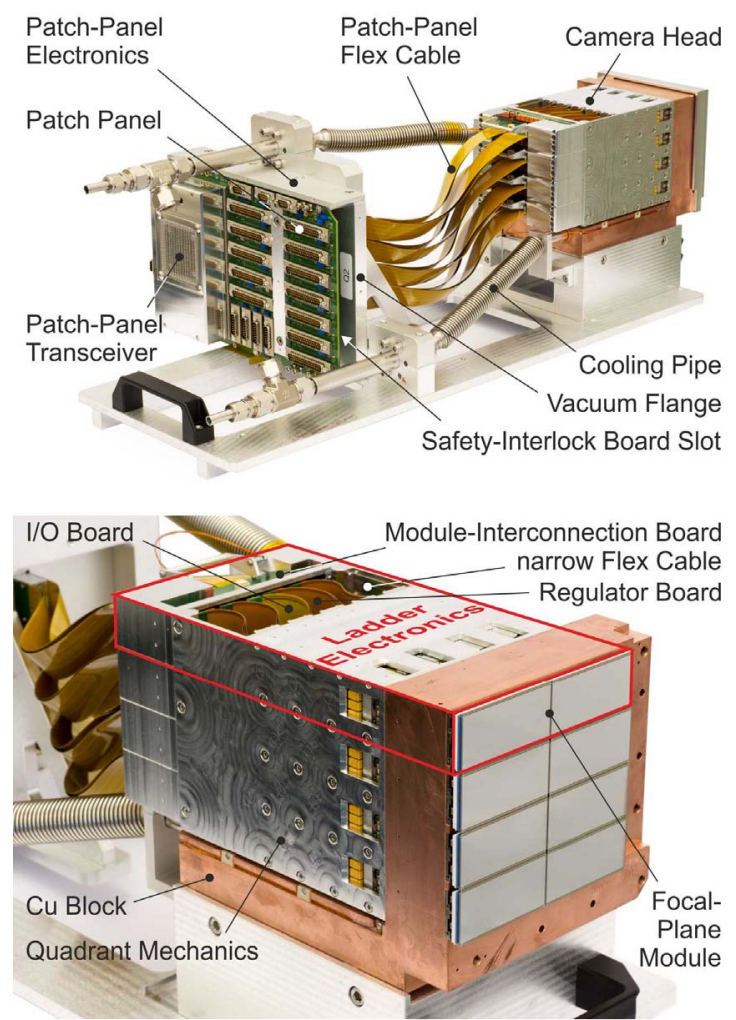

Fig. 1. Photograph of a quadrant of the mini-SDD mega-pixel camera with patch-panel electronics and camera head (top). Flex cables connect the camera head to the patch panel. The lower photograph is a bird's eye view of the camera head with ladder electronics of four FPMs.

ladders with eight monolithic $256 \times 128$ pixel sensors in the focal plane. Five rigid-flex printed-circuit boards (PCBs) and a narrow flex cable are perpendicularly oriented to the FPM. The outermost four PCBs are regulator boards. The central PCB acts as an I/O board. Their flex leads and the narrow flex cable are connected to the module-interconnection board. All six PCBs and the narrow flex cable represent the ladder electronics. As shown in Fig. 1 (top), four further flex-cable pairs (patch-panel flex cable) connect the ladder electronics of the camera head to a large patch-panel PCB. The rigid parts of the flex-cable pairs are glued into corresponding trenches in a vacuum flange. They are $90^{\circ}$-twisted relieving a motion of quadrants. A patch-panel transceiver is mounted on top of the patch panel and processes the data of all four FPMs [7]. The D-sub-connectors on patch panel serve as cable interface to the power crate. On the back side, a safety-interlock board can be inserted (see safety-interlock board slot). This PCB monitors the connectivity to the power channels and protects the quadrant by identifying anomalies and probable hazardous situations in the vacuum chamber [8]. The patch-panel electronics is located outside of the vacuum chamber, the camera head with flex cables inside. The FPMs and quadrant mechanics are fixed to the Cu block. The camera head is cooled through the pipes connecting the $\mathrm{Cu}$ block.

\section{B. Focal-Plane Module}

Fig. 2 shows a photograph of the FPM consisting of a submodule and two bare modules (BM). The submodule is a layer stack of a molybdenum (Mo) frame, a low-temperature co-fired ceramics (LTCC)-based main board (MB), and a Si-heat spreader (for a simplified sketch of the submodule see Fig. 5, left). Five 60-pin connectors and a single 25pin connector on $\mathrm{MB}$ permit the connection of the above mentioned five PCBs and the narrow flex cable, respectively. Both sensors stick out along the long FPM edges. They provide bond-pad rows at both sides, which are wire-bonded to the pad rows on MB's cavity (golden pads). The 1600 wire bonds at a pitch of $150 \mu \mathrm{m}$ connect the BMs. On the back side, two bias voltages per sensor are provided. Both wire-bond pairs are located at the left-hand side of the FPM. Bus lines on the left sensor are used to bias the right one. The step height between pads on MB and sensor is 2.5 and $2.1 \mathrm{~mm}$ for the wire bonds on the front and the back side, respectively. As shown in Fig. 2, different types of adhesives were applied for FPM assembly. An epoxy adhesive is used between frame and MB, whereas a urethane-film adhesive fills the gaps between power dissipating MB components and frame. A large preform of the same type is used between MB and heat spreader. Ag-filled epoxy sheets are embedded between the ASICs and heat spreader. Two alignment pins located at opposite frame corners ensure proper alignment of the frames to the $\mathrm{Cu}$ block.

\section{Choice of Materials and Processes}

The choice of materials concerns the frame, MB, and heat spreader as well as the adhesives that bond the frame and heat spreader to the MB. A force of about $33 \mathrm{~N}$ appears during the insertion of the PCBs into the 60-pin connectors. The 408 devices on MB limit the total bonding area to the frame to $\sim 5 \mathrm{~cm}^{2}$. Every insertion cycle loads the bonding area nearest to the connector (see Fig. 2) to about $0.8 \mathrm{~N} / \mathrm{mm}^{2}$. The camera head will be operated at $\leq 10^{-5}$ mbar and requires adhesives conform to the NASA out-gassing requirements ASTM E595. The high reliability of the gluing contacts is mandatory, because an operating temperature of about $-20^{\circ} \mathrm{C}$ is foreseen, and a warm-up to room temperature could be necessary many times during a lifetime. A second aspect concerns the deformation of the module caused by the different coefficients of thermal expansion (CTE). The frame should exhibit a large Young's modulus and a CTE preferably matching the CTE of the MB material. $\mathrm{Si}$, as heat-spreader material, offers a moderately high thermal conductivity and provides an ideal contact material to the ASIC-back sides of the BMs. The warpage of the module should be minimized in order to have a proper flatness during the following macro-assembly steps of gluing and wire bonding.

\section{A. Adhesives}

A low-modulus adhesive is a preferred choice for bonding parts with higher CTE mismatch, whereas classical epoxies are applicable at low CTE mismatch. For our application, Epoxy Technology's thermally-conductive epoxy of the type EPO-TEK H70E and Aptek Laboratories' low-modulus urethane film adhesive of the type DAT-A-THERM 1303 B/C/B $(\mathrm{B} / \mathrm{C} / \mathrm{B})$ were chosen. The abbreviations $\mathrm{B}$ and $\mathrm{C}$ refer to the 


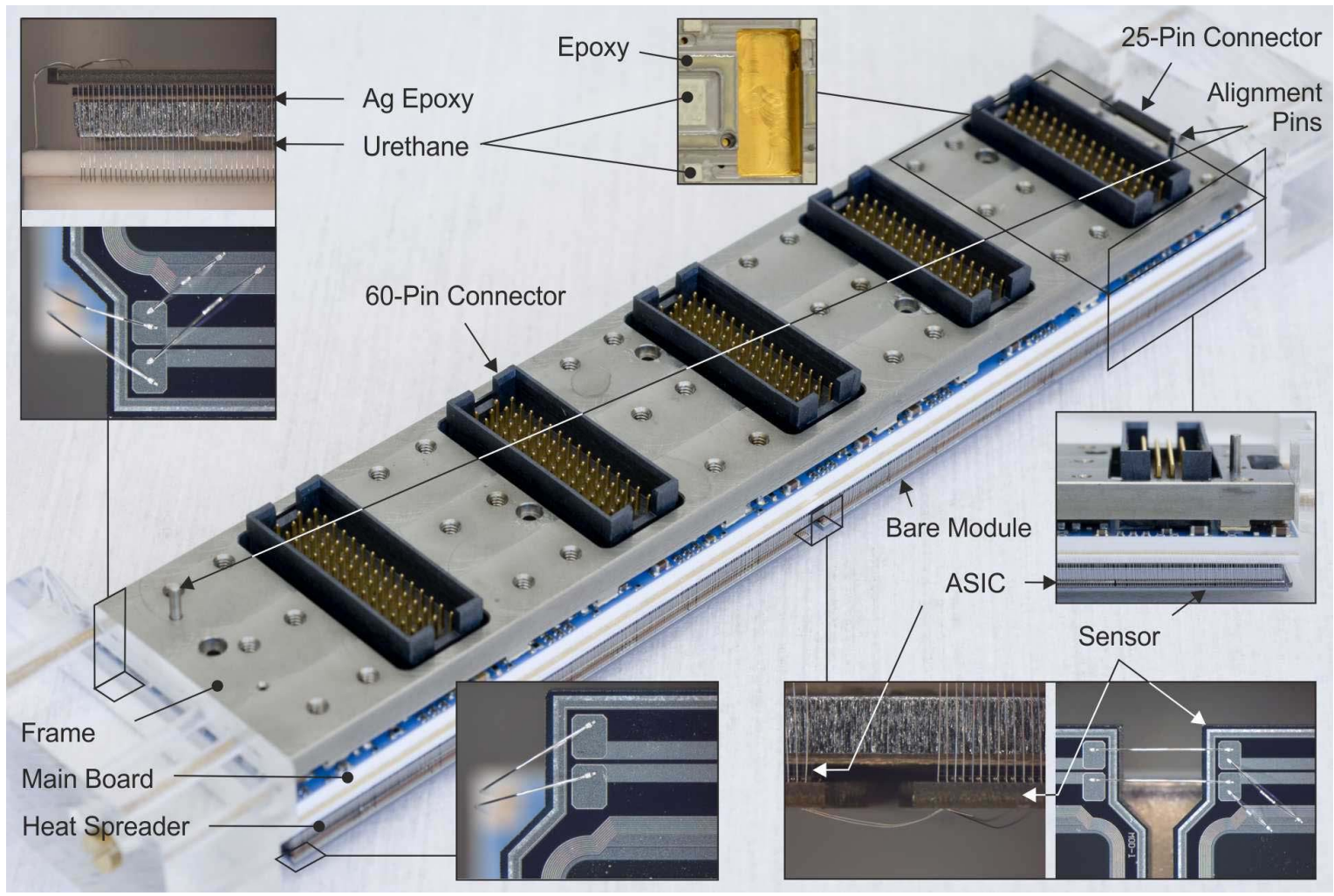

Fig. 2. Photograph of a mini-SDD FPM. Frame, MB, and heat spreader form a submodule. The submodule is mounted on the top of two BMs. Eight ASICs are flip-chip mounted onto a sensor. Wire bonds connect the sensors to the MB.

stage partly cured (epoxy) and fully cured (urethane), respectively. We investigated the fundamental properties of both adhesives using glass substrates. Curing tests were performed under different temperature and pressure conditions. B/C/B was designed so that when the sheet is heated, the viscosity of the B stage will be drastically reduced and when cured under pressure voids will be filled. A side effect is the squeezing out of epoxy. By lowering the temperature and pressure, there will be less squeezing and this may also be sufficient to prevent warping of the package when it is cooled down again from curing temperature to room temperature and later on to $-20^{\circ} \mathrm{C}$ in vacuum. Warping can also be reduced further if the curing temperature is slowly ramped. Several temperature profiles were tested for cross linking of the B/C/B's B-stage epoxy and $\mathrm{H} 70 \mathrm{E}$. In case of $\mathrm{B} / \mathrm{C} / \mathrm{B}$, the curing was performed for $175 \mathrm{~min}$ at $80{ }^{\circ} \mathrm{C}$ and $85^{\circ} \mathrm{C}$ and for $43 \mathrm{~min}$ at $123^{\circ} \mathrm{C}$. The curing of the samples using $\mathrm{H} 70 \mathrm{E}$ was performed for $46 \mathrm{~min}$ at $80^{\circ} \mathrm{C}$. Fig. 3 shows the layer thickness (left $y$-axis) versus contact pressure. In case of $\mathrm{B} / \mathrm{C} / \mathrm{B}$, a pressure of $400 \mathrm{~g} / \mathrm{cm}^{2}$ should be maintained as we observed more large air bubbles when the pressure is reduced, especially at lower curing temperatures. This is illustrated by the right $y$-axis. A treatment below $85{ }^{\circ} \mathrm{C}$ and up to $600 \mathrm{~g} / \mathrm{cm}^{2}$ leads to a reduced epoxyon-glass contact area below $\sim 80 \%$. Best values of $\sim 97 \%$ can be achieved at $400 \mathrm{~g} / \mathrm{cm}^{2}$ for $123{ }^{\circ} \mathrm{C}$. Accordingly, the total $\mathrm{B} / \mathrm{C} / \mathrm{B}$ thickness decreases with increasing pressure at higher curing temperatures. For pressures between 400 and $600 \mathrm{~g} / \mathrm{cm}^{2}$, a B/C/B-layer thickness between 320 and $330 \mu \mathrm{m}$ was reached. In case of H70E, a mean layer thickness of

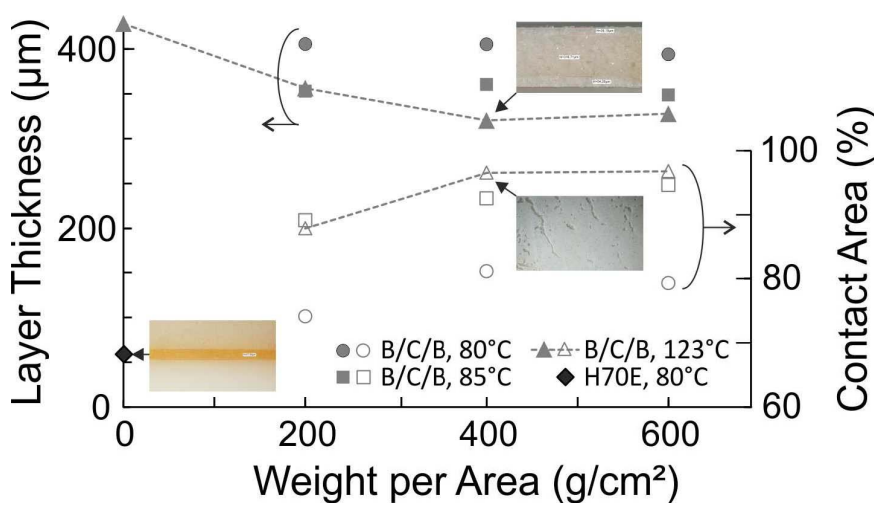

Fig. 3. Layer thickness and contact area versus pressure during curing.

about $58 \mu \mathrm{m}$ was measured. The insets in Fig. 3 show the corresponding cross-sectional views. We can clearly identify the B- and C-stage layers of $\mathrm{B} / \mathrm{C} / \mathrm{B}$ (right, top). The image below is a top view on the B-stage layer tacking the glass surface. The dark structures represent embedded air bubbles.

In summary, the application of $\mathrm{H} 70 \mathrm{E}$ offers lower curing temperatures than $\mathrm{B} / \mathrm{C} / \mathrm{B}$ and allows the lowest pressure. For $\mathrm{B} / \mathrm{C} / \mathrm{B}$, a minimum pressure of $400 \mathrm{~g} / \mathrm{cm}^{2}$ is needed. Taking the thermal conductivity of $\mathrm{H} 70 \mathrm{E}[0.9 \mathrm{~W} /(\mathrm{mK})]$ and $\mathrm{B} / \mathrm{C} / \mathrm{B}$ $[>1.5 \mathrm{~W} /(\mathrm{mK})]$ into account, the thermal resistance of an $\mathrm{H} 70 \mathrm{E}$ layer is more than three times lower than for a $\mathrm{B} / \mathrm{C} / \mathrm{B}$ layer. Therefore, $\mathrm{H} 70 \mathrm{E}$ is the better choice for low CTE mismatch and small contact areas. 


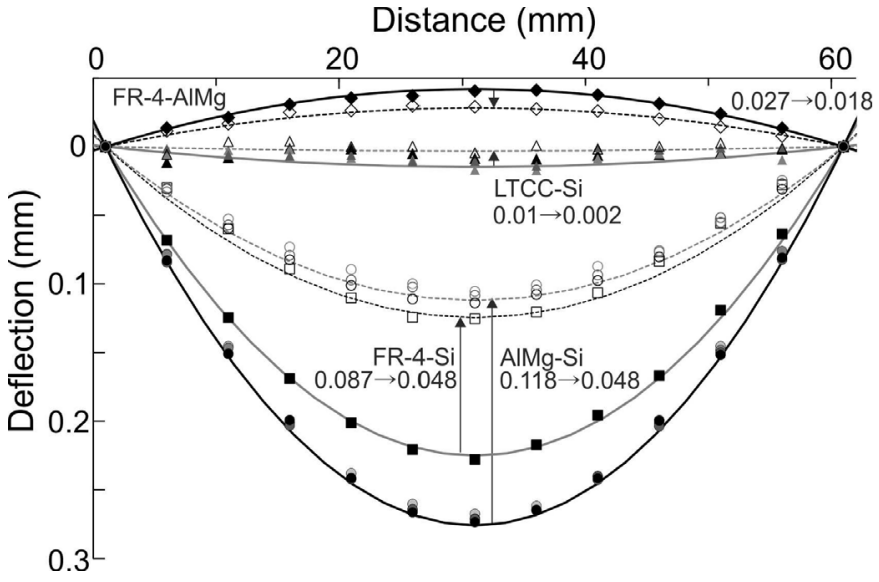

Fig. 4. Measured (symbols) and calculated (curves) deflection of pairs of plates. The arrows mark the reduction of deflection from $\mathrm{H} 70 \mathrm{E}$ samples to $\mathrm{B} / \mathrm{C} / \mathrm{B}$ samples. The misfit-strain numbers are given in percent.

Shear testing is a commonly used method to determine the strength of a die bonded to a substrate. The initial shear force-displacement curve was measured. Within the elastic range, the slope of $\mathrm{H} 70 \mathrm{E}$ amounts to $\sim 180 \mathrm{~N} / \mathrm{mm}$. As expected, B/C/B shows a lower value of $\sim 100 \mathrm{~N} / \mathrm{mm}$. The maximum load force for $\mathrm{H} 70 \mathrm{E}$ and $\mathrm{B} / \mathrm{C} / \mathrm{B}$ translates into a rupture-stress limit of 17.3 and $5.4 \mathrm{~N} / \mathrm{mm}^{2}$, respectively. Both mean values lie well above the $0.8-\mathrm{N} / \mathrm{mm}^{2}$ insertion limit of the 60-pin connectors. No essential degradation was observed for both types of adhesive after thermal stress tests.

\section{B. Deformation}

Deformation studies were needed to determine the efficiency of using $\mathrm{B} / \mathrm{C} / \mathrm{B}$ to compensate for the impact of CTE mismatches. The curvature of a pair of plates bonded together with a uniform misfit strain can be obtained from simple beam-bending theory taking the force and momentum balance into account [9]. Large radii with respect to the plate length lead to an analytical relationship for the deflection height, depending on Young's modulus, thickness, and CTE of the plate materials as well as on the difference between curing and room temperature $(\Delta T)$. The curve depicts a line segment of a circle. Fig. 4 shows the measured deflection for different material sets (symbols). Full symbols represent the use case of $\mathrm{H} 70 \mathrm{E}$, open symbols of $\mathrm{B} / \mathrm{C} / \mathrm{B}$. The solid and dashed lines describe the theoretical curves for $\mathrm{H} 70 \mathrm{E}$ and $\mathrm{B} / \mathrm{C} / \mathrm{B}$, respectively. The efficiency of $\mathrm{B} / \mathrm{C} / \mathrm{B}$ to reduce the CTE mismatch-induced deformation can be easily evaluated by comparing the deflection heights of $\mathrm{B} / \mathrm{C} / \mathrm{B}$ with $\mathrm{H} 70 \mathrm{E}$ samples. The misfit strain $\left(\mathrm{CTE}_{1}-\mathrm{CTE}_{2}\right) \cdot \Delta T$ is maximal for the AlMg-Si pair with $\mathrm{H} 70 \mathrm{E}$ and amounts to $0.118 \%$. $\mathrm{B} / \mathrm{C} / \mathrm{B}$ reduces the misfit strain to $0.048 \%$, equivalent to a CTE-mismatch reduction of $\sim 78 \%$. FR-4 plates were cut out from an $\mathrm{MB}$ sample and bonded to $\mathrm{Si}$ and $\mathrm{Al}-\mathrm{Mg}$ alloy plates. The CTE of FR-4 was extracted by fitting. Very similar values of 17.5 and $18.4 \mathrm{ppm} / \mathrm{K}$ were determined for the FR-4-Si and FR-4-AlMg sample, respectively. With $\mathrm{B} / \mathrm{C} / \mathrm{B}$, the misfit strain was reduced by $\sim 45 \%$ and $\sim 33 \%$ when bonded to $\mathrm{Si}$ and AlMg, respectively. In case of the LTCC-Si samples, a
CTE value of $4 \mathrm{ppm} / \mathrm{K}$ was extracted, which comes very near to $4.5 \mathrm{ppm} / \mathrm{K}$ specified by the vendor. The misfit strain of $0.01 \%$ for $\mathrm{H} 70 \mathrm{E}$ decreases to $0.002 \%$ for $\mathrm{B} / \mathrm{C} / \mathrm{B}$, equivalent to a CTE-mismatch reduction of about $88 \%$. These results demonstrate the high efficiency of strain absorption in $\mathrm{B} / \mathrm{C} / \mathrm{B}$.

\section{Main Board and Frame}

The $\mathrm{MB}$ is an 18-layer LTCC substrate of the size $125.5 \mathrm{~mm} \times 28.2 \mathrm{~mm}$. The total thickness of the PCB is $2.26 \mathrm{~mm}$. We calculated a mean value of the in-plane and vertical thermal conductivity of 12.6 and $3.9 \mathrm{~W} /(\mathrm{mK})$, respectively. The in-plane value is lower than for FR-4 and can be compensated by the Si-heat spreader $[148 \mathrm{~W} /(\mathrm{mK})]$. The CTE of LTCC is very close to the CTE of Si $(2.3 \mathrm{ppm} / \mathrm{K})$.

Altogether, an LTCC-test run comprising five samples, preproduction run with eight samples, and a final production run of thirty samples were carried out. The LTCC-substrate warpage strongly depends on the layout of the metal layers. In the test run, a deflection of about $70 \mu \mathrm{m}$ was found, whereas the second run showed a warpage of around $50 \mu \mathrm{m}$. In the production run, the deflection values range between 187 and $263 \mu \mathrm{m}$ with a mean value of $235 \mu \mathrm{m}$. With a view on the large initial MB warpage, the combination of an LTCC MB with Mo frame is preferable from the mechanical point of view.

Finally, the beam-bending theory was applied to estimate the submodule deflection of the Mo frame/LTCC MB/Si-heat spreader layer stack. In the simulations, the experimentally verified misfit-strain reduction of $\mathrm{B} / \mathrm{C} / \mathrm{B}$ and an operating temperature of $-20{ }^{\circ} \mathrm{C}$ were taken into consideration. The theoretical deflection height was around $6 \mu \mathrm{m}$.

\section{Submodule Assembly}

The Mo frame is placed upside down onto a carrier plate. $\mathrm{H} 70 \mathrm{E}$ is dispensed on stand-off areas located on the frame's $\mathrm{MB}$ side near to the connectors (see Fig. 2, inset top middle). $\mathrm{B} / \mathrm{C} / \mathrm{B}$ pads are placed on further stand-off areas in the MB center as well as along the long frame edges. They thermally connect devices on MB. The alignment is supported by two pins located at opposite corners of the frame and a round and long hole in the $\mathrm{MB}, \mathrm{B} / \mathrm{C} / \mathrm{B}$ sheet and heat spreader. The layer stack is finally covered by another plate and placed inside an oven. A weight of $12.8 \mathrm{~kg}$ provides a pressure of $2.6 \mathrm{~kg} / \mathrm{cm}^{2}$ to the H70E-contact areas and $6.4 \mathrm{~kg} / \mathrm{cm}^{2}$ to the $\mathrm{B} / \mathrm{C} / \mathrm{B}-\mathrm{pad}$ contact areas. The pressure to the $\mathrm{B} / \mathrm{C} / \mathrm{B}$ sheet between $\mathrm{MB}$ and heat spreader amounts to the desired $400 \mathrm{~g} / \mathrm{cm}^{2}$. The curing process in the production run was performed at $125^{\circ} \mathrm{C}$ for $73 \mathrm{~min}$. The ramp up and downtime is $\sim 90 \mathrm{~min}$ and $\sim 10 \mathrm{~h}$, respectively.

\section{Process Evaluation}

\section{A. Submodule}

Fig. 5 summarizes the results of different methods to evaluate the quality of the embedded gluing contacts of the FPM. The optical microscopy image (OMI) shows the Mo frame placed upside down onto the golden carrier plate [Fig. 5(a)]. 


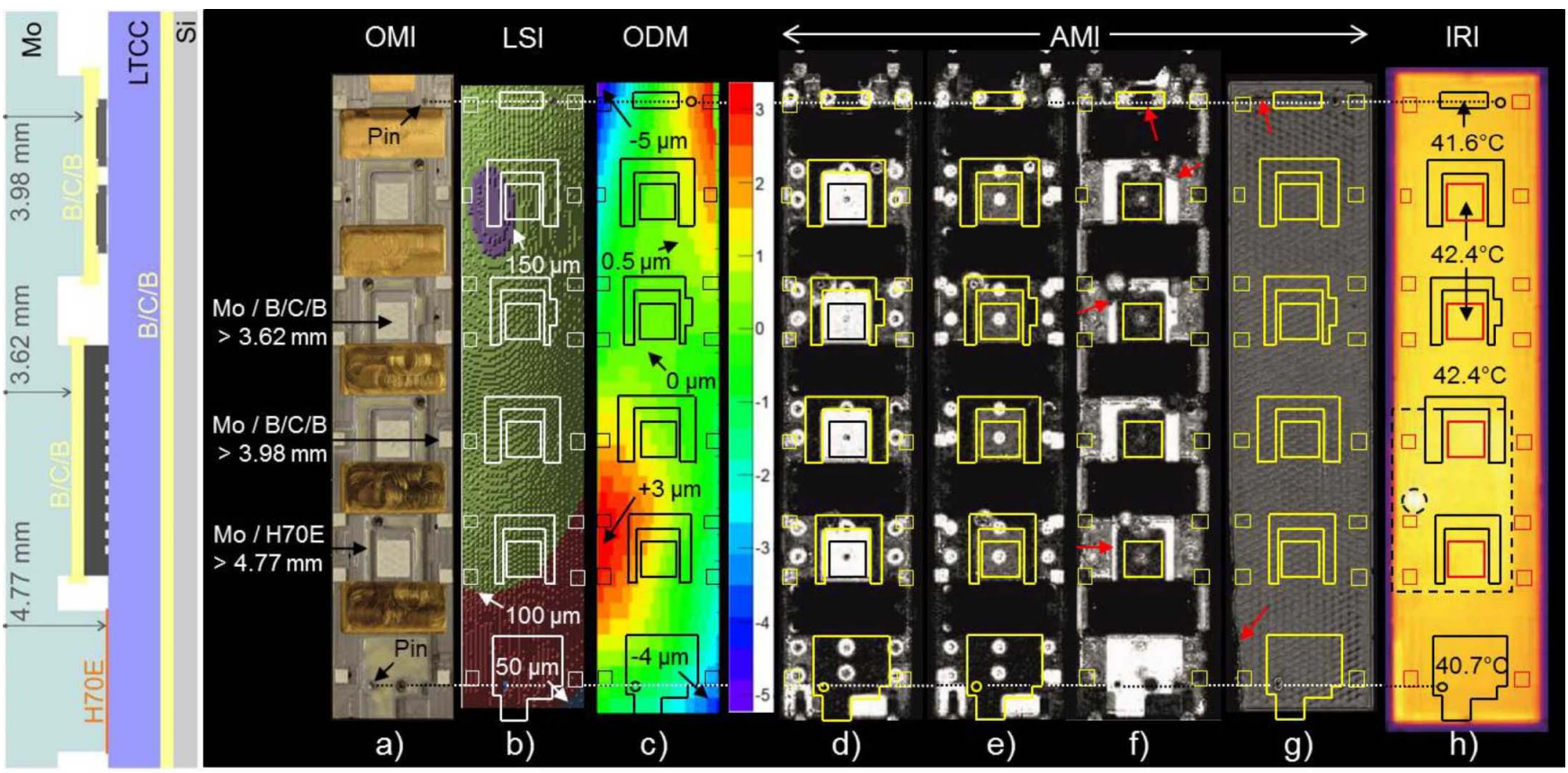

Fig. 5. Simplified cross-sectional view through the submodule (left). (a) H70E was used for the direct contact between Mo frame and LTCC substrate. Large and small square-shaped B/C/B preforms fill the gaps between the frame and active devices as well as between frame and line-termination resistors, respectively. Submodule images are obtained (a) from optical microscopy (OMI), (b) from LSI, (c) from ODM, (d)-(g) from acoustic microscopy (AMI), and (h) from infrared microscopy (IRI).

The distances of the epoxy (yellowish) and B/C/B preforms (white) to the frame's top side are given in $\mathrm{mm}$. The two alignment pins (top right and bottom left) are marked by arrows. The adhesive areas are marked in all images.

The laser-scan microscopy (LSI) image shows the heightsurface profile of the Si-heat spreader at room temperature [Fig. 5(b)]. The numbers mark contour lines in $\mu \mathrm{m}$. Optical deflection measurements (ODM) visualizes the temperature-induced changes in the heat-spreader surface profile [Fig. 5(c)]. The submodule was moderately cooled down from $22.8{ }^{\circ} \mathrm{C}$ to $9.5{ }^{\circ} \mathrm{C}$. Numbers in $\mu \mathrm{m}$ are given for characteristic regions. For regions in the vicinity of direct contact areas (see $\mathrm{Mo} / \mathrm{H} 70 \mathrm{E}>4.77 \mathrm{~mm}$ ), the surface profile remains nearly unchanged (shades of green). Positive height changes were measured in the neighborhood of the upper right alignment pin and in the lower half near to the left heat-spreader edge. Highest negative height changes appear at the upper left and lower right corner. Taking the minimum and maximum values into account, a temperature-induced maximum profile change is $\sim 0.7 \mu \mathrm{m} / \mathrm{K}$.

Acoustic microscopy imaging (AMI) is a nondestructive technique to look inside objects and to detect embedded defects. Ultrasound waves penetrate most materials, and AMI is also sensitive enough for the investigation of interfaces of the adhesive layers. Scans through the maximally 4.77-mm-thick Mo frame were performed, and meaningful intensity images could be taken from Mo-frame side [Fig. 5(d)-(f)]. The four quadratic white areas represent intensity profiles of the waves reflected at the Mo-B/C/B interfaces in $3.62 \mathrm{~mm}$ depth [Fig. 5(d)]. There is no indication of inhomogeneity in this layer. AMI image [Fig. 5(e)] is a profile belonging to Mo-B/C/B interface in $3.98 \mathrm{~mm}$ depth.
All small-format $\mathrm{B} / \mathrm{C} / \mathrm{B}$ preforms are clearly visible. AMI image [Fig. 5(f)] shows the deepest interface in $4.77 \mathrm{~mm}$ depth, where the H70E layer makes the Mo-LTCC contact. Red arrows mark regions with possible gluing gaps (upper three arrows) and a narrow gluing region (lower arrow). We attribute the top right and bottom left red region in the ODM image to the presence of adhesive gaps and the narrow adhesive contact. Further AMI investigations were performed from the submodule-back side through the Si-heat spreader. The visibility of a capillary crack demonstrates the high spatial resolution [red arrow near the top edge in Fig. 5(g)]. The lower arrow marks a chipped region which was unintentionally touched during the laser scanning. The initial rhombic-shaped islands of the B-stage layer remain visible. The structure reflects a remaining inhomogeneity of the mineral filling during gap filling and cross linking. Due to the B/C/B's ability to reduce the misfit strain significantly, the ultrasound waves cause mainly particle motions perpendicular to the propagation direction rather than motions in the propagation direction. Therefore, the $\mathrm{B} / \mathrm{C} / \mathrm{B}$ layer is nearly opaque for this method.

Finally, the submodule was electrically operated in freeconvection mode at room temperature. Infrared images (IRI) were taken from the heat-spreader side. Red boxes in the IR image shown in Fig. 5(h) mark the locations of activated heat sources. During the heat-up phase, the temperature increased continuously and uniformly, indicating that the heat conduction through adhesive contacts into the Mo frame works properly. Some steady-state temperature values are given in degree Celsius. Here, the region in the vicinity of the lower two large red boxes was excluded, because they show a superposition of the object emission and reflections coming from the IR-camera case (dashed rectangle) and IR-camera optics (dashed circle). 
The maximum temperature difference amounts to $\sim 1.7 \mathrm{~K}$. For comparison, a single $\mathrm{MB}$ was also operated under the same conditions, and a maximal temperature difference of $\sim 15.6 \mathrm{~K}$ was measured. The large difference between both steady-state temperature differences demonstrates the high heat-spreading efficiency of the layer construction.

Finally, temperature cycle tests were also performed to the submodule. Altogether, 324 cycles between $-40{ }^{\circ} \mathrm{C}$ and $125{ }^{\circ} \mathrm{C}$ were executed. The module passed all optical inspections as well as the electrical test. In the production run, all 30 submodules were extensively electrically tested using a probe station with customized and commercial measurement equipment. All submodules passed the tests.

\section{B. Bare Module}

The assembly steps of the BM production were performed using a flip-chip bonder of the type FINEPLACER femto. The ASICs were produced in 130-nm CMOS technology and used electroplated SnAg solder. The ASICs were tested at waferlevel and declared as known-good die at less than $1 \%$ pixelfailure occurrence. Before assembly, the solder balls were prereflowed in order to get rid of the needle imprints. The sensor chips use $\mathrm{Cu}$ as under-bump metallization. Sensors with a leakage current below $500 \mathrm{nA}$ at $-150 \mathrm{~V}$ were used for BM production. During processing, the solder pads of the sensor chips were first deoxidized using formic-acid vapor. After this cleaning step, the ASICs were flip-chip mounted onto the sensor at $70{ }^{\circ} \mathrm{C}$ and $10-\mathrm{N}$ force. The ASICs were held in place by a low-melting organic. The final module reflow took place in a separate reflow oven under formic-acid vapor atmosphere at $270{ }^{\circ} \mathrm{C}$. During the whole process, the sensitive sensor-back side was protected by a silicone foil.

Fig. 6(a) shows a typical surface profile of the BM. The $\mathrm{BM}$ warpage is characterized by a maximum deflection around $80 \mu \mathrm{m}$, where the curvature has the same sign as for the submodule's heat spreader [see Fig. 5(b)]. The exemplarily chosen X-ray image of an ASIC-corner section does not show abnormalities at all [see Fig. 6(b)]. Neither voids nor missing connections can be seen. Fig. 6(c) shows a cross section of a solder joint. Cu-containing phases are visible at both bumppad interfaces. A homogeneous wetting of the $\mathrm{Cu}$-solder pads occurred and no cracks or voids are visible.

Altogether, $47 \mathrm{BMs}$ were produced. For BM selection, the ASIC and leakage-current tests were repeated. The BM test identified five BMs with ASIC and sensor failures. These BMs were rejected for further FPM assembly.

\section{Focal-Plane Module}

The assembly of twenty FPMs was executed. The process starts with the BM placement onto a silicone foil on a base plate of sufficient size for two BMs. The placement was again carried out using the flip-chip bonder. Fiducials on sensor chips were utilized for precise alignment of the BMs to each other. The base plate was then placed in a customized fixture, and two 120- $\mu$ m-thick Ag-filled epoxy sheets of the type ABLEFILM ECF561E were put on top of the ASIC-back sides of the BMs. Due to the usage of preformed sheets, any soaking

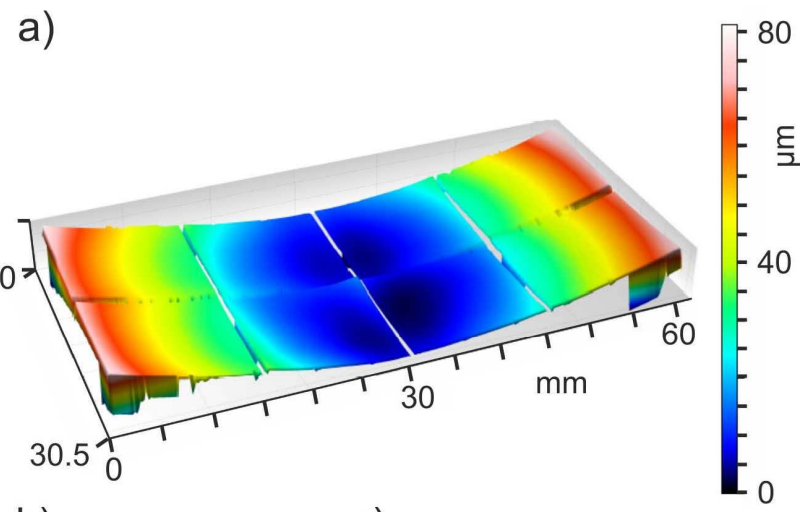

b)

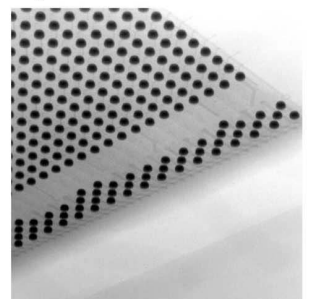

c)

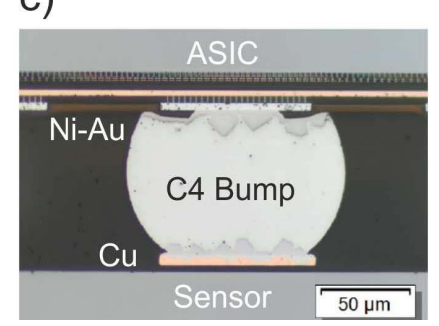

Fig. 6. (a) Surface profile of a BM, (b) X-ray image of an ASIC-corner section, and (c) microphotograph of a solder joint in a cross-sectional view. All solder joints do not show abnormalities and are of good quality.

of adhesive can be avoided and the ASIC-back sides are fully covered by adhesive. Afterward, the submodule was precisely placed with the help of stoppers located at all four module sides. The upper part of the fixture was used to apply a constant force between about 100 and $150 \mathrm{~N}$ to the frame during the curing phase performed in a conventional oven at $150{ }^{\circ} \mathrm{C}$.

In order to validate the alignment accuracy of the BMs with respect to each other and with respect to the frame, all FPMs were geometrically measured. Fig. 7 shows the specification of measurement points and methods to determine the in-plane displacement and rotation of the sensors as well as their $z$ displacement and tilting. Fourteen survey points were specified, eight corner pads on sensor 1 ( $\mathrm{P} 0, \mathrm{P} 1, \mathrm{P} 6$, P7) and sensor 2 (P2, P3, P4, P5) as well as two diagonal alignment-pin coordinates (P8, P9) and four corner points on frame (P10, P11, P12, P13). P8 and P9 correspond to the round- and long-hole positions of the Cu block, respectively. The four corner points were taken as reference points for all $z$ values. The corresponding $x, y$, and $z$ values were determined optically by a coordinate-measurement system. The FPMs were aligned in a way that the outermost wire-bond pads on both sensors (P0 and $\mathrm{P} 3$ ) represent the $x$-axis at $y=0$, where the origin of the Cartesian coordinates is represented by the upper left corner of the outermost left pad of sensor 1 (P0). P8 defines the end position of an FPM with respect to the $\mathrm{Cu}$ block. The $y$ coordinate of $\mathrm{P} 9$ with respect to $\mathrm{P} 8(\Delta Y)$ defines the in-plane rotation of the frame. The known positions of the corner pads of both sensors allow the determination of measurement error. The standard deviation amounts to $5.6 \mu \mathrm{m}$ and limits the measurement precision.

P2 and P0 specify the displacement of the two BMs to each other. Fig. 8(a) shows the corresponding misalignments 


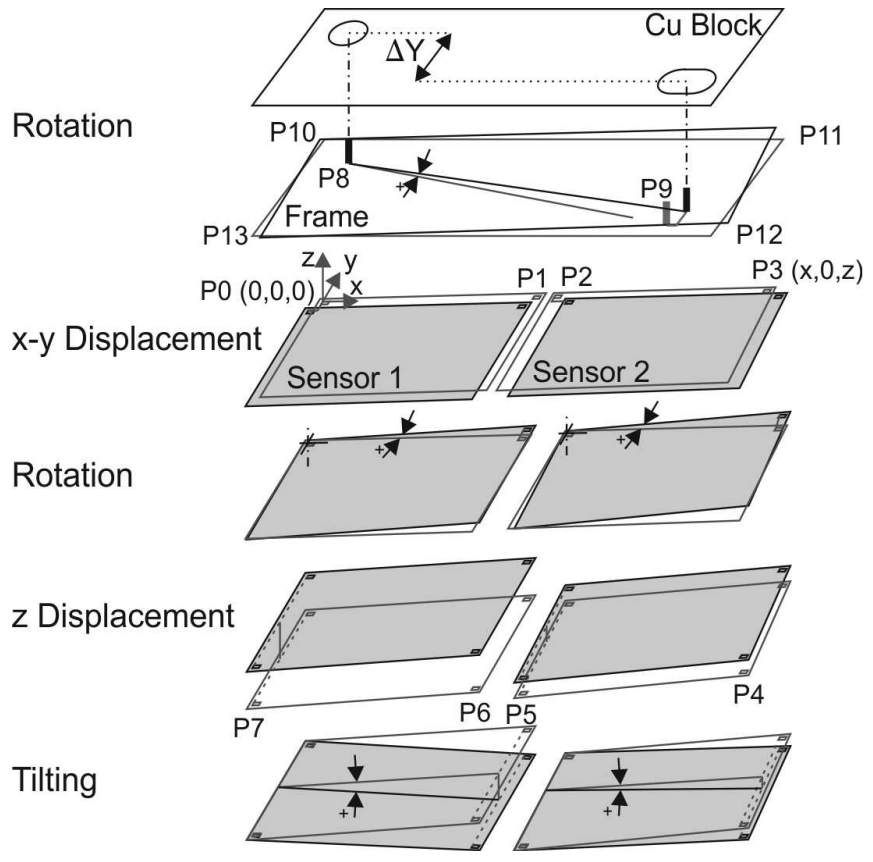

Fig. 7. Schematic illustrating the positions of measurement point on frame (P8, P9), sensor 1 (P0, P1, P6, P7) and sensor 2 (P2, P3, P4, P5) as well as the instruction for the determination of sensors' in-plane displacements and rotations, their displacements in $z$-direction and their tilting.

in the $x$ - and $y$-direction (open symbols). The three outliers stem from the assembly-optimization phase using the first three FPMs. Excluding these outliers, the right BM (Fig. 7, sensor 2) is systematically displaced by 10.5 and $-5.1 \mu \mathrm{m}$ in the $x$ - and $y$-direction, respectively. The standard deviation amounts to $9.5 \mu \mathrm{m}$ in the $x$-direction and $5 \mu \mathrm{m}$ in the $y$-direction. $\mathrm{P} 0$ and $\mathrm{P} 8$ represent the basis for the determination of the displacement of the BMs with respect to the submodule frame (fully symbols). The left BM (Fig. 7, sensor 1) is systematically displaced by $-12.8 \mu \mathrm{m}$ in the $x$-direction and $-4.9 \mu \mathrm{m}$ in the $y$-direction. The standard deviation is 61.8 and $64.4 \mu \mathrm{m}$ in the $x$ - and $y$-direction, respectively.

Fig. 8(b) summarizes the deviation from target values for $\mathrm{P} 9$ with respect to $\mathrm{P} 8$ in the $y$-direction. On the $x$-axis, the positions of alignment holes in the frame are plotted. The mean value and standard deviation of the deviation from the desired distance of holes are -0.6 and $6.2 \mu \mathrm{m}$, respectively. The $y$-axis refers to the corresponding pin positions. The pin-related mean value and standard deviation amount to 8.5 and $75.3 \mu \mathrm{m}$, respectively. The large spread of $\Delta Y$ of about $\pm 150 \mu \mathrm{m}$ dominates the rotational error of the focal plane and confirms possible collision conflicts during quadrant mounting. The rotational error of the BMs themselves is in the order of several micrometers.

After submodule assembly, line scans were performed along the long axis of the heat spreader. The step height between the outermost right and left value at the short-edge sides represents the tilting. These points were also used for leveling. The maximum value of the leveled curve was then taken as deflection height. Both values were plotted on the $x$-axis in Fig. 8(c). The tilting values spread between -32 and $139 \mu \mathrm{m}$ and show a mean value of $36.7 \mu \mathrm{m}$, whereas the
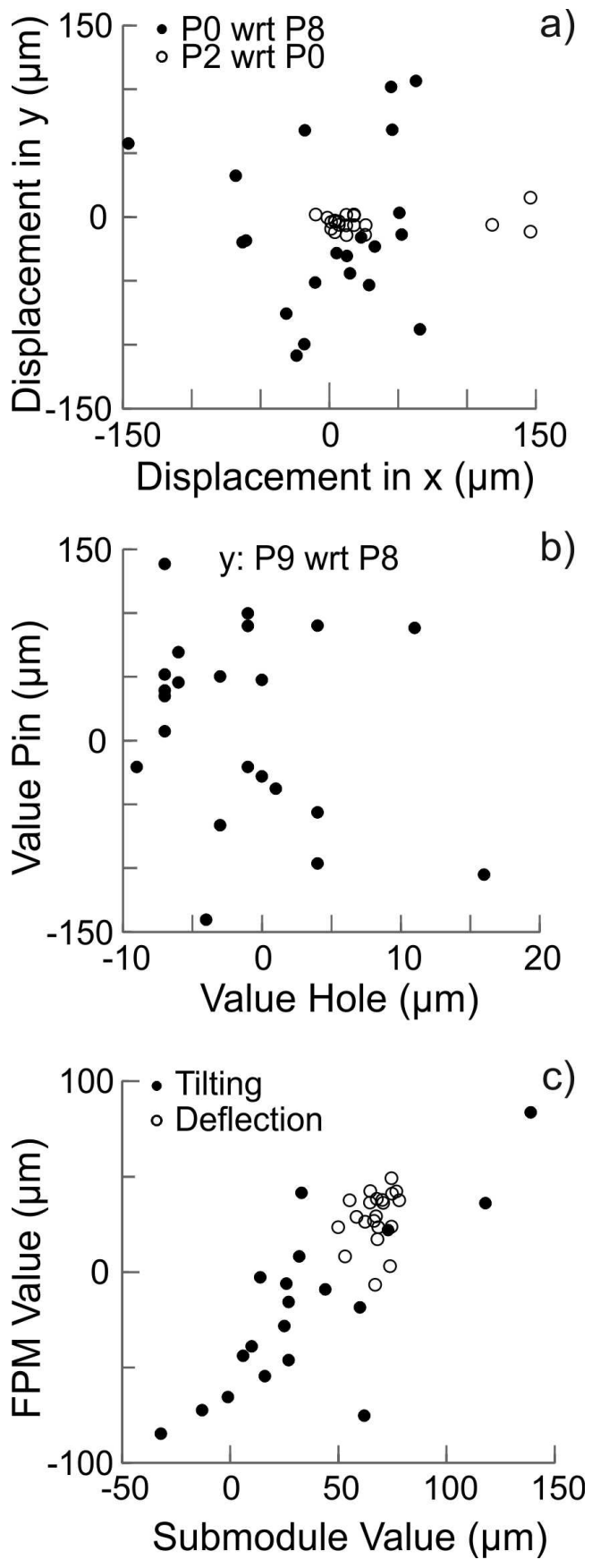

Fig. 8. (a) In-plane displacement of the BM and FPM, (b) in-plane rotation of the frame, and (c) tilting and deflection of the FPMs in comparison to the submodules.

deflection varies between 50 and $78.1 \mu \mathrm{m}$ with a mean value of $67.1 \mu \mathrm{m}$. This value is about one order of magnitude larger than the estimated value from the simple beam-bending theory. We attribute this deviation to the initial LTCC-MB warpage. The results of the FPM tilting and deflection is plotted on the $y$-axis in Fig. 8(c). The FPM heights at the module sides were calculated from the difference of the mean $z$ values of corner points (P0/7, $\mathrm{P} 3 / 4)$ and the mean $z$ values on the frame top (P10/13, P11/12), where the height difference represents the FPM tilting. The FPM deflection arises from the mean $z$ value $\mathrm{P} 2 / 5$ referred to the corresponding mean $z$ value on 
the frame top side, which was interpolated from P10 to P13. The tilting values vary between -84.7 and $83.6 \mu \mathrm{m}$ with a mean value and standard deviation of -22.6 and $42.5 \mu \mathrm{m}$, respectively. The FPM deflection values are within the range -6.7 to $49.2 \mu \mathrm{m}$ and show a mean value of $28.6 \mu \mathrm{m}$ and a standard deviation of $13.7 \mu \mathrm{m}$. Compared to the mean deflection value of the submodule's heat spreader of $67.1 \mu \mathrm{m}$, the final deflection of the FPM's sensor layer is reduced by more than a factor of two. We attribute this improvement to the flexibility of the rubberized Ag-epoxy sheets between the heat spreader and BMs. In any case, the deformed BMs [see Fig. 6(a)] nestle to the deformed heat spreaders, and the soft silicone foil compensates the inhomogeneous contact pressure during the final FPM-gluing step at least partly.

The final process step in the FPM production is the wirebonding. An Al wire of $25 \mu \mathrm{m}$ diameter was used. A mean pull force of $9.3 \mathrm{cN}$ was obtained with heel-crack failure code on sensor and MB pads. The large step height of the backand front side wire bonds ensure their reduced sensitivity on LTCC shrinking and displacements between sensors and MB.

\section{Quadrant Mounting}

The mounting process starts with the placement of four FPMs into a quadrant case. The risk of this step arises from the small nominal vertical distance between the sensors of neighboring FPMs of only $257 \mu \mathrm{m}$. Collisions were avoided by a careful allocation of FPMs taking their individual displacements and rotations into account (see Fig. 8). The FPMs were screwed to the $\mathrm{Cu}$ block (see screw threads in Fig. 2), and the narrow flex cables were connected to the 25-pin connectors. Afterward, all subassemblies of the ladder electronics were plugged in and screwed to the quadrant mechanics. Finally, the flex-cable pairs were connected to the camera head.

\section{Characterization}

\section{A. Sensor Leakage}

During flip-chip mounting and macro assembly, the sensorback side was protected by a silicone foil. Both processes were performed at low forces in order to reduce the mechanical load stress and impact on ASIC functionality and sensorleakage current. The room-temperature leakage currents were measured at $-150-\mathrm{V}$ backside voltages. At the front side, the charge-collecting anode of the pixel cell is enclosed by the two drift rings. The voltages of the inner and outer ring were set to -20 and $-25 \mathrm{~V}$, respectively. The voltage gradient forces the signal electrons radially inwards to the anode.

The mean value of the chip-level leakage current is about $309.3 \mathrm{nA}$, which decreased to $233.1 \mathrm{nA}$ after flip-chip mounting. We attribute this decrease to annealing effects caused by the thermal processes during reflow. The FPM-level currents lie almost above the BM values. The $95 \%$ of the assembled sensors remain below $32 \mu \mathrm{A}$ with a mean value of $\sim 0.8 \mu \mathrm{A}$, and $92.5 \%$ remain below $5 \mu \mathrm{A}$ with a mean value of $\sim 0.7 \mu \mathrm{A}$. Below 1- $\mu \mathrm{A}$ leakage current, $77.1 \%$ of the modules fall into this class and have a mean value of $415 \mathrm{nA}$.

A detailed noise-performance analysis was executed for our reference FPM [10]. The total mean value of $\sim 86$ electrons was found. This value is much larger than the contribution of the leakage current. The sensor leakage will be smaller at the foreseen operating temperature of $-20{ }^{\circ} \mathrm{C}$ so that all FPMs were accepted for quadrant integration from the sensor-leakage point of view. We conclude that all assembly steps from flipchip bonding to FPM mounting up to quadrant integration do not lead to significant sensor malfunctions.

\section{B. Functionality}

After completion of the FPM assembly, each FPM was connected to its PCBs and first installed into a vacuum-test stand to test the full functionality. The operation under vacuum condition was chosen in order to hold the LTCC MBs free of electromigration caused by high electric fields. The reference FPM of the series production is in operation for many months and was also operated at $-20^{\circ} \mathrm{C}$ during beam times [10]. From this experience, we derived the following tests as essential to select robust FPMs. The tests comprised the dark-image data taking for default settings, an ADC gain and offset trimming, a front-end trimming with on-chip injection circuitry, and a final test of the full signal chain with spot illuminated sensors at default front-end gain settings. All tests must be carried out at full speed of $4.5 \mathrm{MHz}$, and all ASICs must be fully operated and read out using all 800 storage cells at a repetition rate of 10-Hz. The front-end electronics and ADCs were powered for about $200 \mu \mathrm{s}(\geq 800 / 4.5 \mathrm{MHz})$ at the given repetition rate.

First, the front-end amplifiers were operated in buffer mode, and images with zero input were taken (dark images). This test reveals immediately fundamental supply and readout failures. Afterward, the on-chip DAC was used to perform an ADC-gain trimming targeting $320 \mathrm{LSB} / \mathrm{V}\left(2^{8} / 0.8 \mathrm{~V}\right)$. This test confirms a proper and stable chip initialization and programming of the pixel registers through a JTAG chain and verifies the achievability of the trimming precision of around 2\%. Fig. 9(a) shows the gain maps of all FPMs of the series production. The FPMs no. 1 to 16 were finally selected for integration. Number 17 is our reference FPM, which passed all tests, but reveals the highest displacement. Number 18 shows malfunctions of 68 pixels just beyond our $1 \%$ class limit for two ASICs (marked in red). FPM no. 19 passed all tests but showed an increased ring current and a single ASIC, which could not be gain-trimmed temporarily. Number 20 passed the dark-image test but could not be programmed via JTAG in a daisy chain. We attribute this defect to a missing interconnection on BM.

For the fully or partly working ASICs, the gain spread of all nineteen FPMs is in very good accordance with the designed granularity. Twelve ASICs show artifacts (areas in the dark blue or multicolor). Six of them are fully affected and six only partly. Two of the partly affected ASICs (no. 12 and 14) show error codes (no ADC-comparator trigger) for only 20 pixels at the end of a single column and still fall into known-good die class. Nine columns of the faulty ASIC of no. 11 could not be trimmed properly. In case of FPM no. 13, the faulty ASIC showed a very large number of pixels with the error-code occurrence and unstable memory content. The occurrence of this malfunction is much less pronounced without biasing the front end and completely vanishes with 
a)

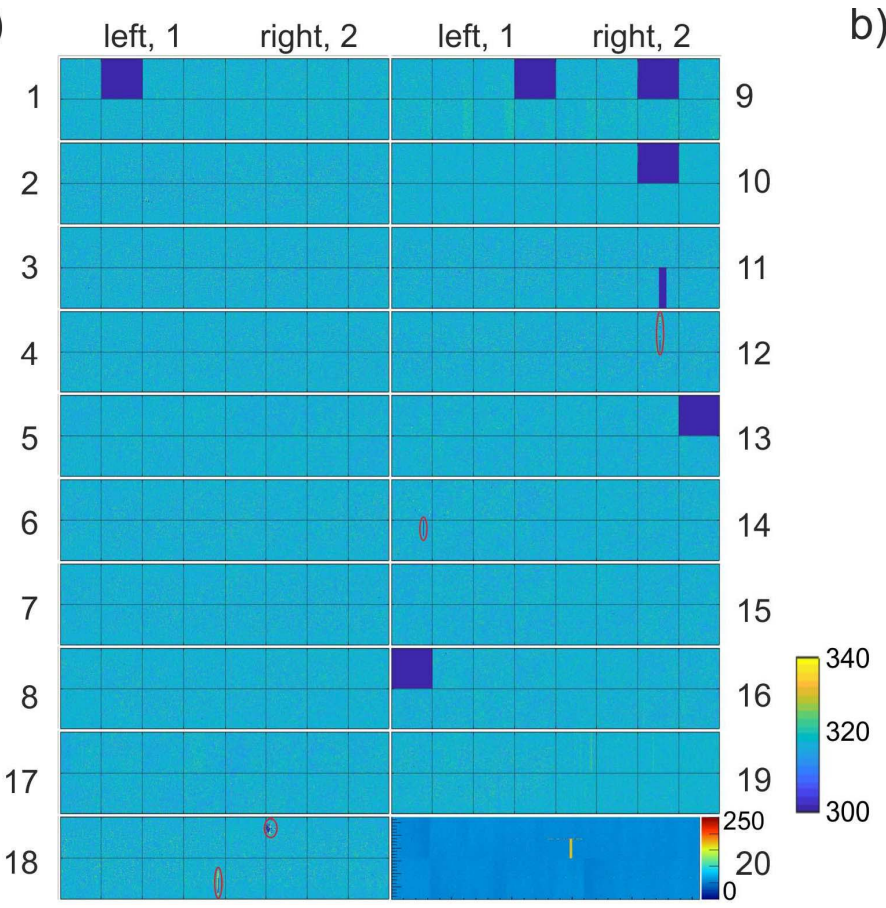

b)

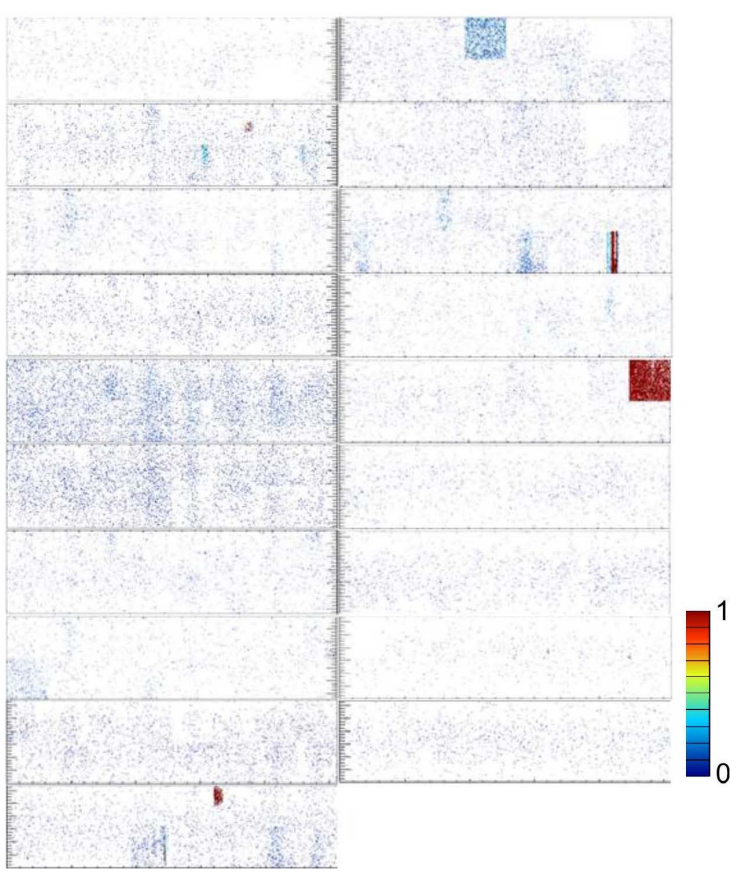

Fig. 9. Maps of the (a) ADC gain and (b) offset after trimming of 19 FPMs. For FPM number 20, a dark image is shown instead. The unit of the gain-color code is LSB/V, and the unit of the dark-image color code is LSB. The offset maps represent the standard deviation of the offset distribution. The unit is LSB.

$25 \%$ down-powered pixels and with front-end biasing. During ASIC wafer-level and BM test, only one-fourth of the pixels were activated simultaneously, so that such a failure could not be detected. That trimming failures occur at the column end and trimming success depends on the number of activated pixels indicates a power-related problem. The faulty ASIC of FPM no. 16 is also affected by a power problem as well as the left faulty ASIC of FPM no. 9. Its faulty ASIC on the right-hand side and the faulty ASIC of FPM no. 10 are most probably caused by a phase-alignment problem between a clock and data channel. The ASIC malfunction of FPM no. 1 is caused by a damaged wire-bond pad.

Furthermore, the performance of the offset trimming was determined. The purpose of this trimming step is to place the entries of dark images into the center of an ADC bin (720-ps bin size). Deviations from this optimum position can be caused by the limited 4-bit trimming granularity ( $\sim 60 \mathrm{ps}$ ), by noise as well as by deviations from the nominal bin size [4], [10]. Any distribution across more than a single bin leads to a standard deviation different from zero. Therefore, this test is very sensitive to deviations from normal circuit behavior. Fig. 9(b) shows the maps of the standard deviation in LSB. The numbering scheme is identical to Fig. 9(a). White areas represent the optimum case with entries in a single bin (0 LSB) except for the ASICs which failed the gain-trimming test. Brown pixels represent the situation, where $67 \%$ of the entries fall into a single bin (1 LSB). The $98.2 \%$ of the pixels have a standard deviation of less than $0.1 \mathrm{LSB}$. This test result confirms our findings shown in Fig. 9(a). On the other hand, some FPMs show a higher density of colored points over the whole sensor area or in local regions, which was not visible in the previous gain-trimming test. Several of these FPMs were set up with sensors from a batch showing an increased via resistance. Nevertheless, both test results confirm that the ladder trimming can reach the same accuracy level as for our reference FPM.

Finally, all FPMs were illuminated with light in the central region between sensors 1 and 2. The LED spot had a diameter of about $1 \mathrm{~cm}$. This simple test was performed with a single light pulse during data taking of 800 frames at a repetition rate of $10-\mathrm{Hz}$. In this way, one can easily check if both sensors are sensitive to light and if the signal in each pixel is detected at the same time. As in the offset-trimming approach, the RMS values of the pixel histograms are evaluated. Therefore, this test is also sensitive in the regions outside of the LED spot. All installed FPMs passed this test as our reference module did [10].

Altogether, eight of twenty FPMs (40\%) exhibit a malfunction almost caused by a single chip, and more than two-thirds of the sensors of the FPMs have a maximum leakage current below $1 \mu \mathrm{A}$. The majority of these failures are associated with the FPM-gluing step. In case of the ASIC failures, ASICs located near to short edges of the FPMs are mainly concerned, where tilting- and deflection-related mechanical stress is higher than in the center region of the FPM. For future runs, doubling the production of FPMs could compensate the loss due to ASIC failures, sensor leakage, displacements, and handling.

\section{CONCLUSION}

The series-production results of the FPMs of the DSSC mega-pixel X-ray imager are presented. We describe the multi-layer construction of the module and introduce a lowmodulus urethane adhesive to efficiently reduce the misfit 
strain and minimize the module warpage. All setup processes up to the integration of the modules into the quadrant cooling blocks are discussed. The precision of module alignment allows a safe integration and the achieved electrical module performance measures up to our expectations.

\section{ACKNOWLEDGMENT}

The authors would like to thank FhG-ISiT, Itzehoe, Germany, for the acoustic microscopy images, and A. Mussgiller from DESY, Hamburg, Germany, for the optical deflection measurements (ODM) image.

\section{REFERENCES}

[1] M. Porro et al., "Development of the DEPFET sensor with signal compression: A large format X-ray imager with mega-frame readout capability for the European XFEL," IEEE Trans. Nucl. Sci., vol. 59, no. 6, pp. 3339-3351, Dec. 2012.

[2] S. Aschauer et al., "First results on DEPFET active pixel sensors fabricated in a CMOS foundry-A promising approach for new detector development and scientific instrumentation," J. Instrum., vol. 12, Nov. 2017, Art. no. P11013.
[3] F. Erdinger et al., "The DSSC pixel readout ASIC with amplitude digitization and local storage for DEPFET sensor matrices at the European XFEL," in Proc. IEEE NSS/MIC Conf. Rec., Oct. 2012, pp. 591-596.

[4] K. Hansen, C. Reckleben, P. Kalavakuru, J. Szymanski, and I. Diehl, "8-bit 5-MS/s analog-to-digital converter for pixel-level integration," IEEE Trans. Nucl. Sci., vol. 60, no. 5, pp. 3843-3851, Oct. 2013.

[5] K. Hansen, M. Randall, S. Schleitzer, and C. Gutt, "System-level simulation of a X-ray imager with nonlinear gain and per-pixel digitizer: XPCS case study," Nucl. Instrum. Methods Phys. Res. A, Accel. Spectrom. Detect. Assoc. Equip., vol. 613, no. 2, pp. 323-333, Feb. 2010.

[6] K. Hansen, H. Klär, and D. Müntefering, "Camera head of the DSSC X-ray imager," in Proc. Conf. Rec. IEEE Nucl. Sci. Symp. (NSS/MIC), Oct. 2011, pp. 1713-1717.

[7] T. Gerlach et al., "The DAQ readout chain of the DSSC detector at the European XFEL," in Proc. IEEE NSS/MIC Conf. Rec., Nov. 2011, pp. 156-162.

[8] S. Nidhi, H. Klaer, K. Hansen, M. Turcato, M. Kuster, and M. Porro, "Safety-interlock system of the DSSC X-ray imager," in Proc. IEEE NSS/MI/RTSD Conf. Rec., Nov. 2016, pp. 1-4.

[9] S. Timoshenko, "Analysis of bi-metal thermostats," J. Opt. Soc. Amer., vol. 11 , no. 3, pp. $233-255,1925$.

[10] P. Kalavakuru et al., "Performance test of focal-plane modules of the DSSC X-ray imager," presented at IEEE NSS/MIC/RTSD, Nov. 2018. [Online]. Available: https://www.eventclass.org/contxt_ieee2018/onlineprogram/session?s=N-07\#e1486 